\title{
In Situ Liquid SEM Studies of Electrochemical and Radiolytic Processes
}

\author{
Rolf Møller Nielsen ${ }^{1}$, Silvia Canepa ${ }^{1}$, Murat Nulati Yesibolati ${ }^{1}$, Christoffer P. Nielsen ${ }^{2}$, Henrik Bruus ${ }^{2}$, \\ Hongyu Sun ${ }^{1}$ and Kristian Mølhave ${ }^{1}$ \\ 1. Dept. of Micro- and Nanotechnology, Technical University of Denmark (DTU), Lyngby, Denmark. \\ 2. Dept. of Physics, Technical University of Denmark (DTU), Lyngby, Denmark.
}

A custom built system using microfabricated membrane chips as windows to enable liquid phase scanning electron microscopy, Fig 1 [1], provides a number of opportunities compare the liquid TEM systems currently available. Commercial reference electrode and large counter electrode can be inserted and exchanged, flow is possible through connected tubes, and the overall design provides a larger reservoir and hence more controlled flow and diffusion kinetics than the available liquid TEM cells. The SEM resolution cannot compete with TEM, but in electrochemical liquid phase imaging often the TEM resolution is not much better than SEM due to thick liquid layers in the TEM system.

Determining the radiolytic influence of the electron beam on a liquid sample is essential for all liquid phase electron microscopy studies, but so far only modelling has given indication of the chemical species involved [2]. Given the full beam current injected into the liquid cell during SEM, with only minimal backscattering and the overall radiation dose rate can be adjusted to match that of a TEM experiment as in the model study, we have used the electroanalytical abilities of our SEM system to quantify the radiolytically produced species by chronopotentiometry. Ex-situ injection of hydrogen into the electrolyte $\left(0.1 \mathrm{M} \mathrm{H}_{2} \mathrm{SO}_{4}\right)$ gives a rapid drop in the chronopotentiometric voltage reading of a $\mathrm{Pt}$ microelectrode vs. a silver/silver chloride reference electrode. Irradiating the electrode or in the vicinity of the electrode with the electron beam gives a drop in potential, the depends on the beam current, Fig 2, that enables estimation of the hydrogen evolution and also excludes the possibility of any significant hydrogen peroxide evolution that would limit the potential drop. The experiment demonstrates how the SEM system can be used for reliable electroanalytical studies and quantification of radiolytic products.

The well-defined diffusion boundary conditions in the reservoir also enables detailed studies of dendritic growth at over potential limited currents, where diffusion limitations are important boundary conditions. Electrodeposition of copper from $\mathrm{CuSO}_{4}$ solution makes is possible to study the time evolution and structure of dendrites correlated with potentiostatic measurements, Fig 3. Our dendritic growth observations are compared with good qualitative agreement to finite element models of the same process Fig 3 [3].

The SEM system hence provides a reliable and electrochemically controlled platform for detailed quantitative studies of complex electrochemical processes and in some ways complements the capabilities of the TEM liquid cell systems [4].

References:

[1] E. Jensen et al, Ultramicroscopy 129 (2013), p. 63.

[2] N.M. Schneider et al, J. Phys. Chem. C 118 (2014), p. 22373.

[3] C. P. Nielsen and H. Bruus, Phys. Rev. E 92(4) (2015), p. 042302.

[4] The authors acknowledge funding DTU and from the Danish Research Council for Technology and Production Case No. 12-126194 

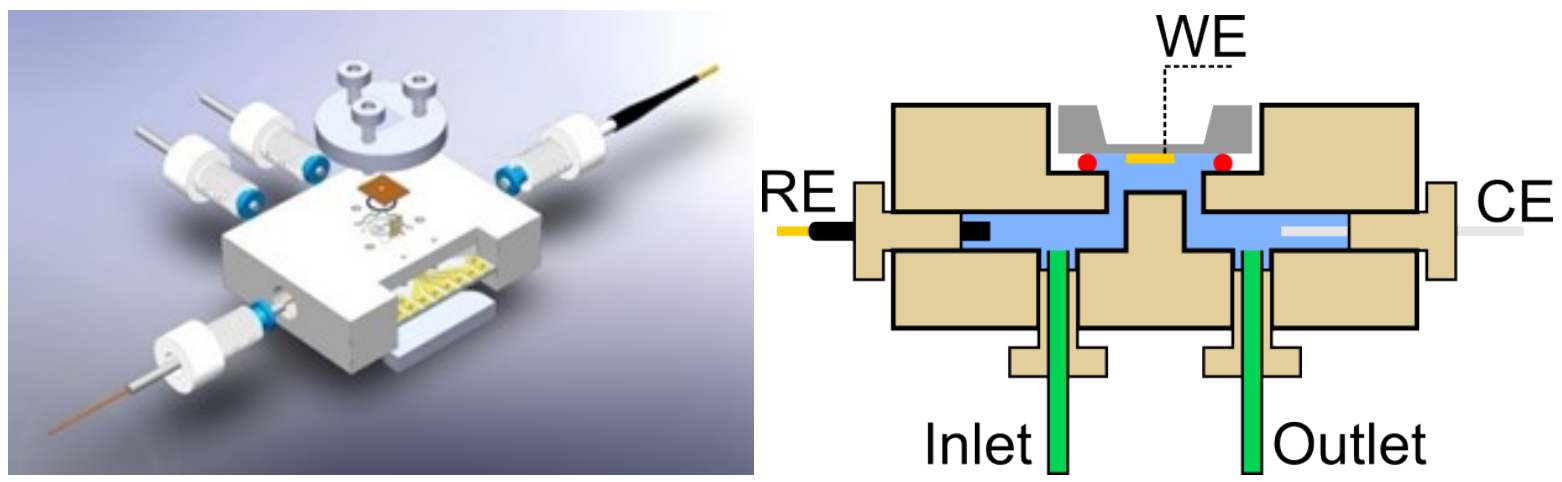

Figure 1. Schematic and diagram of a custom built SEM liquid cell system with exchangeable counter (CE) and reference electrodes (RE) and Platinum working electrodes on a microfabricated exchangeable silicon nitride membrane chip.

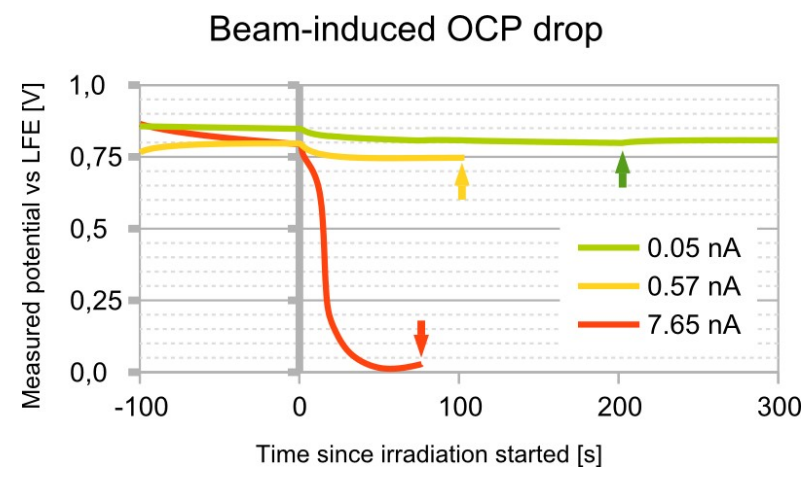

Figure 2. The radiolytic species created by the electron beam causes a distinct drop in the chronopoteniometric signal, reaching that of an solution with at least $25 \%$ saturation of $\mathrm{H}_{2}$.
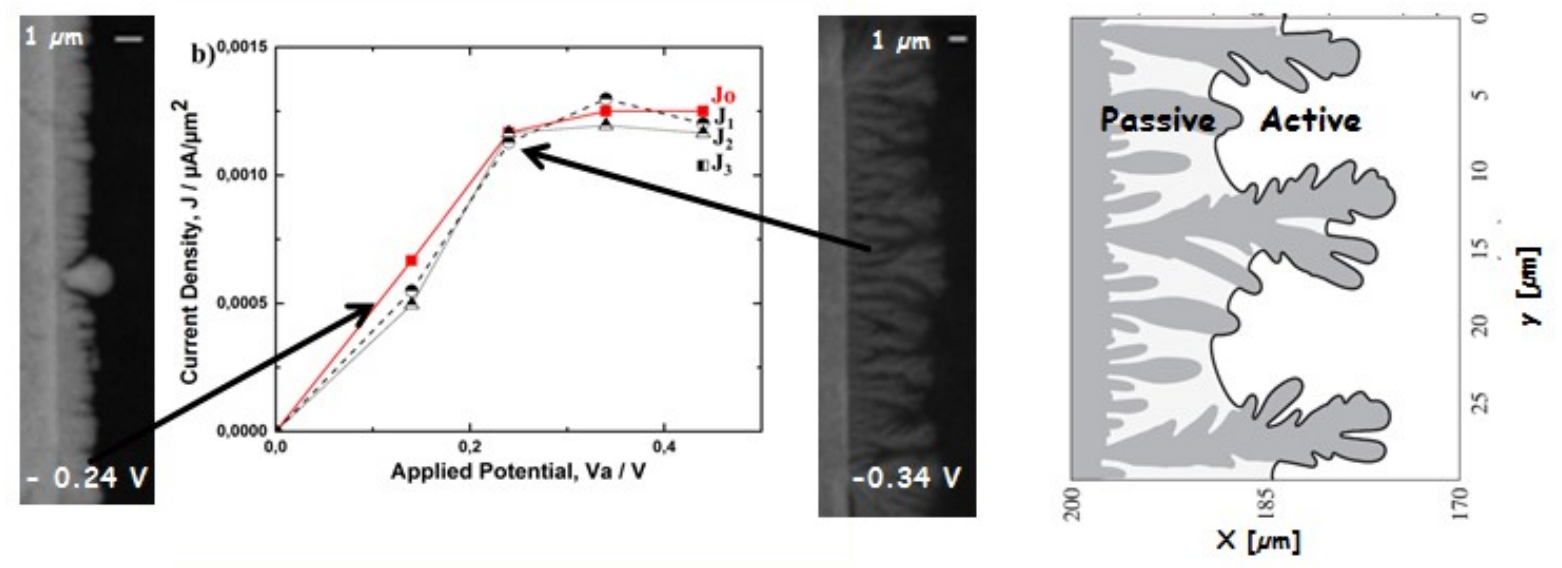

Figure 3. Dendritic copper growth as function of applied potential vs a $\mathrm{Cu}$ reference electrode, shows a plateau reaching the limiting current density as the transition to dendritic growth. The observations are comparable to results obtained by finite element models of the process [3]. 